$<$ Supporting Information $>$

\title{
MSC-Encapsulating In Situ Cross-linkable Gelatin Hydrogels To Promote Myocardial Repair
}

Chan Woo Kim ${ }^{\dagger}$, Chan Joon Kim*, Eun-Hye Park ${ }^{\dagger}$, Seung Bae Ryu ${ }^{\S}$, Yunki Lee ${ }^{\S}$, Eunmin $\mathrm{Kim}^{\dagger}$, Kwonyoon Kang ${ }^{\dagger}$, Kwan Yong Leell, Eun-Ho Choo ${ }^{\dagger}$, Byung-Hee Hwang ${ }^{\dagger}$, Ho-Joong Yoon $^{\dagger}$, Ki Dong Park ${ }^{* *}$, Kiyuk Chang ${ }^{\dagger}$

${ }^{\dagger}$ Division of Cardiology, Department of Internal Medicine, Seoul St. Mary’s Hospital, College of Medicine, The Catholic University of Korea, Seoul 06591, Republic of Korea

$\ddagger$ Division of Cardiology, Department of Internal Medicine, Uijeongbu St. Mary’s Hospital, College of Medicine, The Catholic University of Korea, Uijeongbu 11765, Republic of Korea ${ }^{\S}$ Department of Molecular Science and Technology, Ajou University, Suwon 16499, Republic of Korea

॥ Division of Cardiology, Department of Internal Medicine, Incheon St. Mary's Hospital, College of Medicine, The Catholic University of Korea, Incheon 21431, Republic of Korea

\section{* Correspondence to Chan Joon Kim, MD, PhD}

E-mail: godandsci@catholic.ac.kr

\section{** Correspondence to Ki Dong Park, PhD}

E-mail: kdp@ajou.ac.kr 
Figure S1. Elastic modulus $\left(G^{\prime}\right)$ and viscous modulus $\left(G^{\prime \prime}\right)$ of GH hydrogels with different concentration of $\mathrm{H}_{2} \mathrm{O}_{2}$ in the presence of $\mathrm{HRP}(0.006 \mathrm{mg} / \mathrm{mL})$. Rheological analysis of $(\mathrm{A}) \mathrm{GH}-$ compliant (0.07 wt \% $\left.\mathrm{H}_{2} \mathrm{O}_{2}, 1300 \mathrm{~Pa}\right)$ and (B) GH-stiff $\left(0.14\right.$ wt \% $\left.\mathrm{H}_{2} \mathrm{O}_{2}, 5300 \mathrm{~Pa}\right)$ hydrogels, respectively.

A

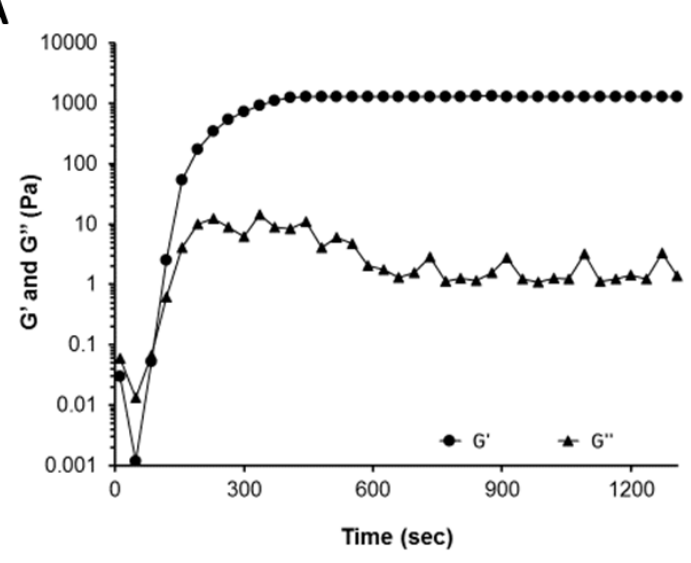

B

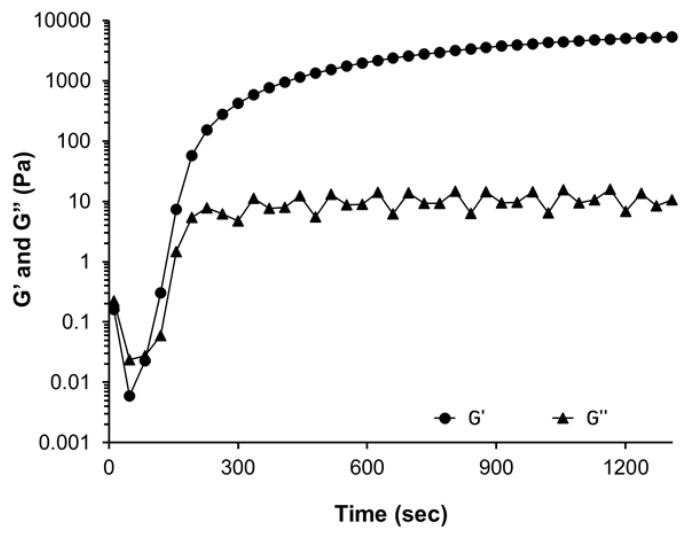


Figure S2 Video. In vitro 3D live/dead staining of MSCs within GH hydrogels. (A) Extensive proliferation and multicellular 3D structure of encapsulated MSCs within the GH-compliant hydrogel. (B) Rounded and less-spread morphologies of encapsulated MSCs within the GHstiff hydrogel.

Figure S3 Video. Echocardiography of MI induced mice at day 28 post-transplantation with PBS, MSCs, gel, or MSCs/gel, respectively.

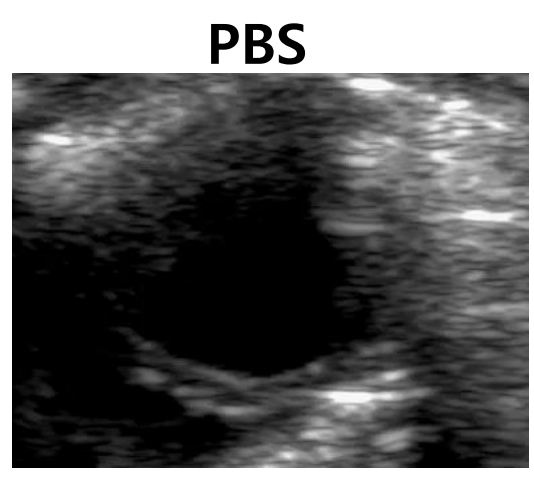

MSCs

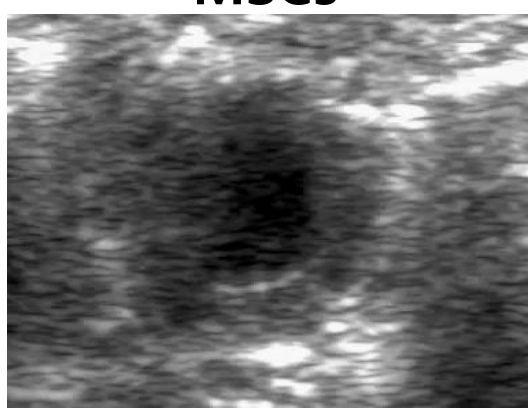

Gel

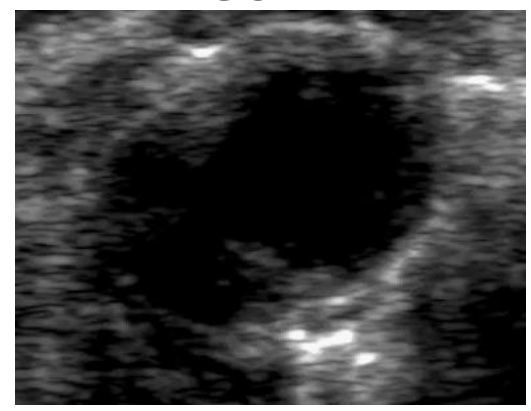

MSCs/gel

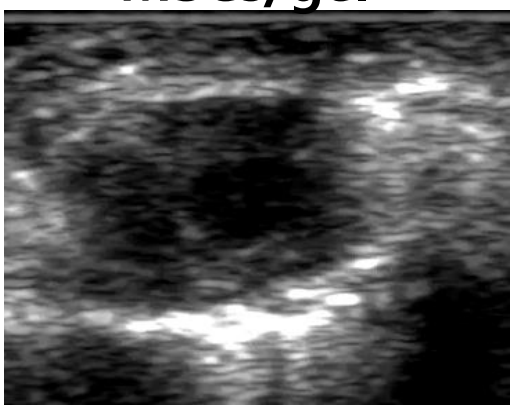


Figure S4. Degradation properties of GH hydrogels for 28 days. (A) In vitro degradation profiles of GH-compliant and GH-stiff hydrogels with or without collagenase $(0.5 \mu \mathrm{g} / \mathrm{mL})$ treatment $(n=3)$. (B) In vivo degradation profiles of the GH-compliant hydrogel labeled with FITC in MI induced mice $(n=3)$.

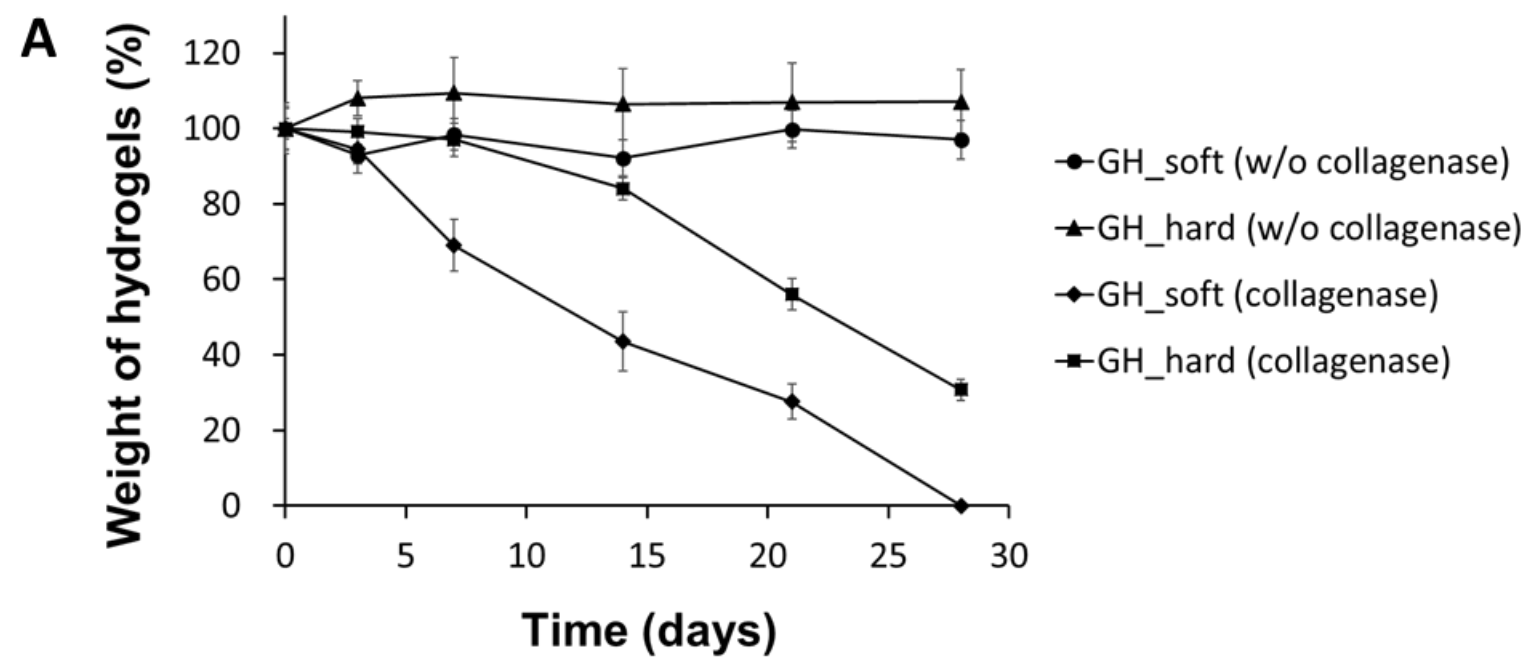

B

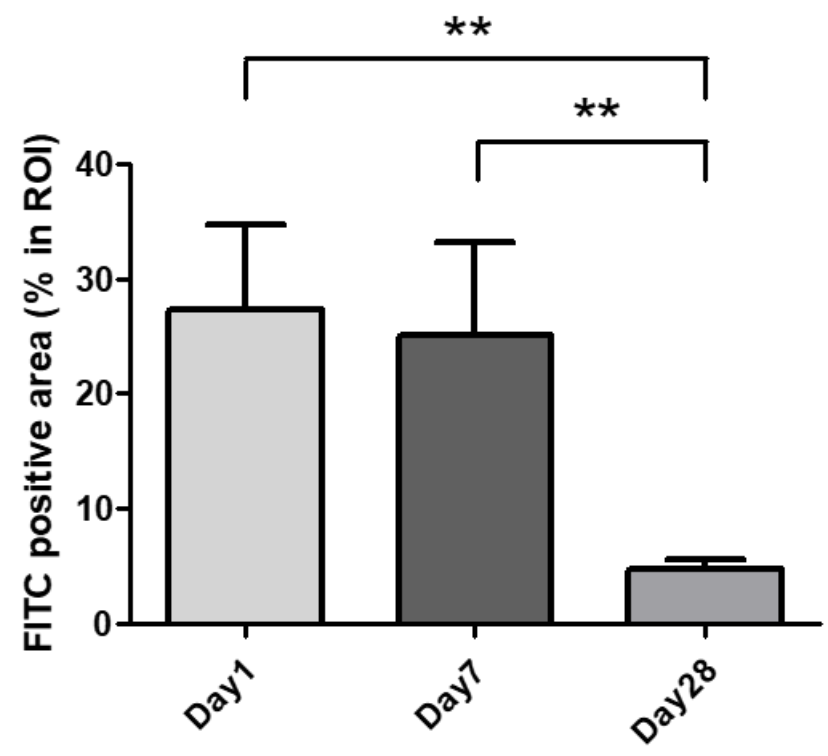

